\title{
Three-Dimensional Properties of Coronal Mass Ejections from STEREO/SECCHI Observations
}

\author{
E. Bosman • V. Bothmer • G. Nisticò • A. Vourlidas • \\ R.A. Howard · J.A. Davies
}

Received: 15 November 2011 / Accepted: 4 September 2012 / Published online: 16 October 2012

(C) The Author(s) 2012. This article is published with open access at Springerlink.com

\begin{abstract}
We identify 565 coronal mass ejections (CMEs) between January 2007 and December 2010 in observations from the twin STEREO/SECCHI/COR2 coronagraphs aboard the STEREO mission. Our list is in full agreement with the corresponding SOHO/LASCO CME Catalog (http://cdaw.gsfc.nasa.gov/CME_list/) for events with angular widths of $45^{\circ}$ and up. The monthly event rates behave similarly to sunspot rates showing a three- to fourfold rise between September 2009 and March 2010. We select 51 events with well-defined white-light structure and model them as three-dimensional (3D) flux ropes using a forwardmodeling technique developed by Thernisien, Howard and Vourlidas (Astrophys. J. 652, $763-773,2006$ ). We derive their 3D properties and identify their source regions. We find that the majority of the CME flux ropes $(82 \%)$ lie within $30^{\circ}$ of the solar equator. Also, $82 \%$ of the events are displaced from their source region, to a lower latitude, by $25^{\circ}$ or less. These findings provide strong support for the deflection of CMEs towards the solar equa-
\end{abstract}

The Sun 360

Guest Editors: Bernhard Fleck, Bernd Heber, and Angelos Vourlidas

Electronic supplementary material The online version of this article (doi:10.1007/s11207-012-0123-5) contains supplementary material, which is available to authorized users.

E. Bosman $(\varangle) \cdot$ V. Bothmer

Institute for Astrophysics, University of Göttingen, 37077 Göttingen, Germany

e-mail: ebosman@astro.physik.uni-goettingen.de

V. Bothmer

e-mail: bothmer@astro.physik.uni-goettingen.de

G. Nisticò

Dipartimento di Fisica, Universita della Calabria, 87036 Rende, Italy

e-mail: giuseppe.nistico@ fis.unical.it

A. Vourlidas · R.A. Howard

Spaces Sciences Division, Naval Research Laboratory, Washington, DC 20375, USA

J.A. Davies

Rutherford Appleton Laboratory, Chilton Oxfordshire, OX11 0QX, UK

e-mail: jackie.davies@stfc.ac.uk 
tor reported in earlier observations, e.g. by Cremades and Bothmer (Astron. Astrophys. 422, $307-322,2004)$.

Keywords Coronal mass ejection

\section{Introduction}

Since the start of science operations in January 2007, the imagers and coronagraphs of the Sun-Earth Connection Coronal and Heliospheric Investigation (SECCHI) suite (Howard et al., 2008), aboard the twin STEREO spacecraft (Kaiser et al., 2008), have provided simultaneous observations of coronal mass ejections (CMEs) from different vantage points in space. Using the white-light synoptic movies provided by the two STEREO/SECCHI/COR2-A and -B coronagraphs, we have compiled a list of 565 coronal mass ejections (CMEs) between January 2007 and December 2010. The CMEs were observed under increasing spacecraft separation angles ranging from about $0^{\circ}$ in the early mission phase up to $175^{\circ}$ in December 2010. The CME list contains basic information, such as Carrington Coordinates of both spacecraft, CME detection times and position angles, etc. and is available online at the website http://soteria-event.uni-graz.at/. ${ }^{1}$ The list was compiled as part of the EU FP7 project SOTERIA (SOlar TERrestrial Investigations and Archives).

A comparison of the monthly average CME rate from the SOTERIA COR2 CME list with the CME rate derived from the SOHO/LASCO CME Catalog ${ }^{2}$ yields a very good correspondence for $\mathrm{CME}$ events with angular widths greater than or equal to $45^{\circ}$. Thus, the SOTERIA COR2 CME list consists of classic large-scale CMEs, such as analyzed, e.g., by Cremades and Bothmer (2004). Figure 1 shows the comparison of the monthly CME rates from SECCHI and LASCO between January 2007 and December 2010, together with the monthly smoothed sunspot number (SSN) provided by the Solar Influences Data Analysis Center (SIDC) ${ }^{3}$ of the Royal Observatory of Belgium. Figure 1 shows that the monthly $\mathrm{CME}$ rates and monthly smoothed sunspot numbers show generally similar trends but not detailed correlations as has been reported in earlier studies (e.g., St. Cyr et al., 2000). We note that both the CME and sunspot monthly rates rise by a factor of three to four between September 2009 and March 2010 and remain high in the following months. This increase can be interpreted as the start of the rise of solar activity towards the next solar maximum expected around 2012-2013. It is interesting to note that the CME rate remains constant (at 10/month for SECCHI and 7/month for LASCO) for several months in 2009 although the corresponding sunspot number hovers around zero. We investigate the low-coronal source regions of these CMEs using the SECCHI Extreme Ultraviolet Imager (EUVI) at 195 and $304 \AA$. We find that they relate to bipolar photospheric regions of lower magnetic flux and quiescent prominence eruptions, in agreement with the results obtained for the CME source regions studied by Cremades and Bothmer (2004). However, for a number of CMEs, no source region could be identified as in the case of the "stealth CME" reported by Robbrecht, Patsourakos, and Vourlidas (2009). The differences between the CME rates and sunspot

\footnotetext{
${ }^{1}$ Also available in Tables 1 and 2 in the Electronic Supplementary Material.

${ }^{2}$ CDAW Data Center, Solar Physics Laboratory (Code 671) Heliophysics Science Division, NASA / Goddard Space Flight Center, Greenbelt Maryland, USA: 1996-2011, SOHO/LASCO CME Catalog, http://cdaw.gsfc.nasa.gov/CME_list/.

${ }^{3}$ Solar Influences Data Analysis Center, Royal Observatory of Belgium: 2010, Monthly and monthly smoothed sunspot number, http://sidc.oma.be/sunspot-data/.
} 


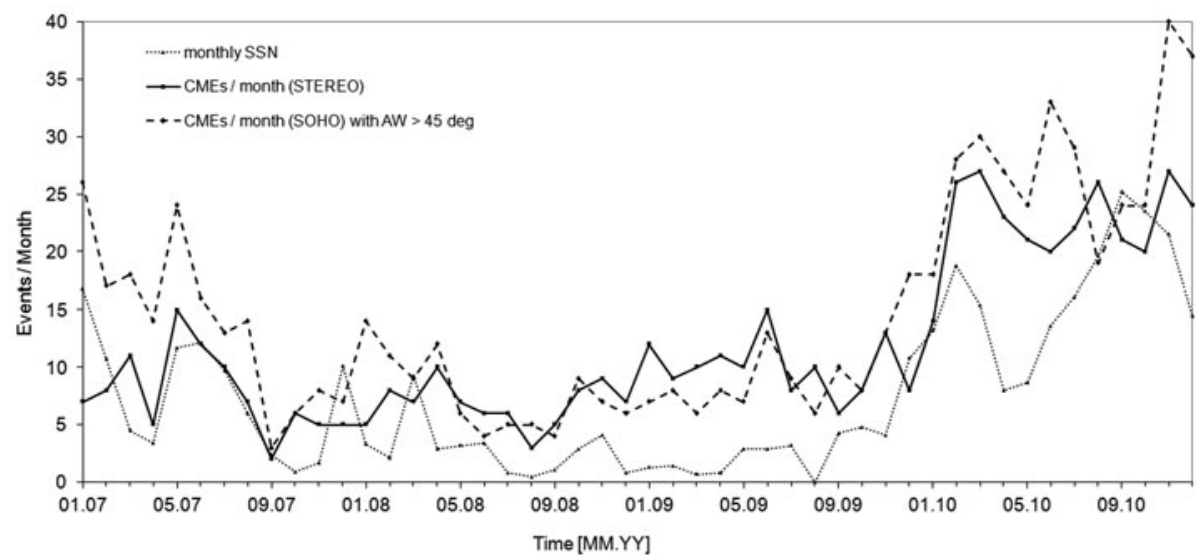

Figure 1 Monthly CME rates observed by STEREO/SECCHI/COR2 (solid line) and those derived from the SOHO/LASCO/C2 CME catalog (dashed line) with an angular width $\geq 45^{\circ}$ for the time period January 2007 until December 2010. The monthly sunspot number (dotted line) is provided by the SIDC at the Royal Observatory of Belgium. (Solar Influences Data Analysis Center, Royal Observatory of Belgium: 2010, Monthly and monthly smoothed sunspot number, http://sidc.oma.be/sunspot-data/.)

numbers after January 2010 can be explained in terms of decaying active regions of less intense magnetic flux remaining unidentified as sunspots but remaining a source of $\mathrm{CME}$ origin, again in agreement with what has been proposed by Tripathi, Bothmer, and Cremades (2004).

From the SOTERIA COR2 list of 565 events, we constructed a "Best-of" list of 120 events based on their clear morphology (judged visually) in the COR2 images. So far, we have fitted 51 of these events as flux ropes with a forward-modeling technique developed by Thernisien, Howard, and Vourlidas (2006) and Thernisien, Vourlidas, and Howard (2009). The flux rope structure is represented by a geometrical construction, called the Graduated Cylindrical Shell (GCS) and is based on the idea that the flux rope morphology can account for the CME white-light observation (Chen et al., 1997; Vourlidas et al., 2000; Cremades and Bothmer, 2004).

In the following sections we give a brief introduction to the GCS Model and a brief presentation of the modeling results and comparisons with the CME source region characteristics.

\section{The GCS Model}

For the investigation of the three-dimensional (3D) structure of the "STEREO/SECCHI/ COR2 Best-of CMEs"4 the Graduated Cylindrical Shell forward-modeling technique developed by Thernisien, Howard, and Vourlidas (2006) was applied. The geometry and electron density distribution of the GCS flux rope geometry is shown in Figure 2. The GCS geometry consists of two funnel-shaped legs each of length $h$. The segment $h$, along the axis through the center of the shell (dash-dotted line), is defined by the center of the Sun, labeled "O",

\footnotetext{
${ }^{4}$ The complete "Best-of" CME list is shown in Table 3 in the Appendix and in Table 3 in the Electronic Supplementary Material.
} 
Figure 2 The Graduated Cylindrical Shell Model with a face-on view on the left and an edge-on view on the right. The assumed electron density distribution is shown in the upper right and described with a Gaussian-like function (Thernisien, Howard, and Vourlidas, 2006).

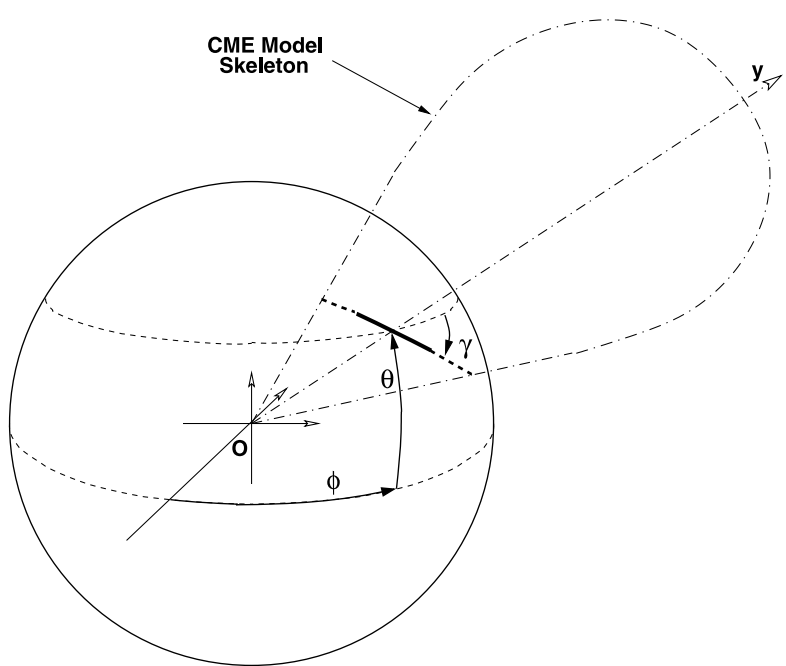

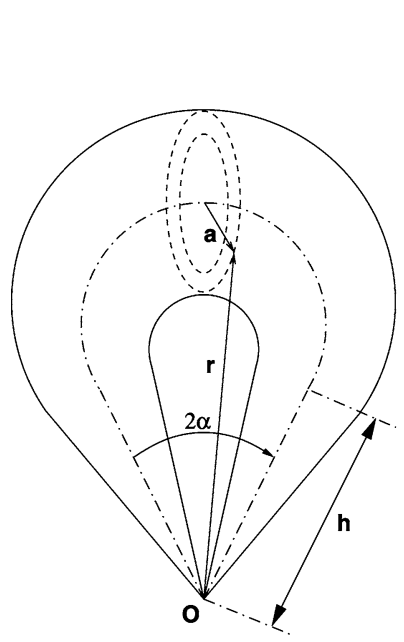

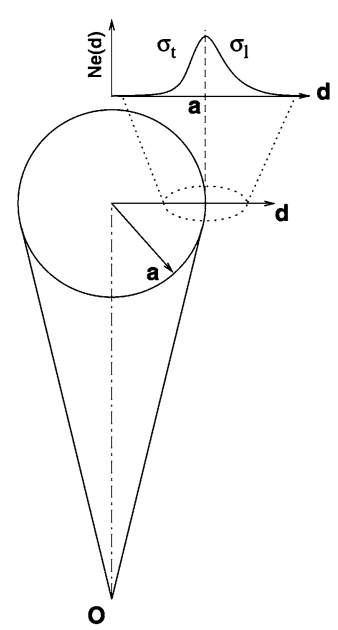

Figure 3 Position and orientation of the GCS Model in 3D Space with the parameters $\phi, \theta$ and $\gamma$ for the Carrington longitude and heliographic latitude of the apex and the tilt angle, respectively (Thernisien, Vourlidas, and Howard, 2009). 
Table 1 Parameters of the GCS Model and the electron density distribution, adapted from Thernisien, Howard, and Vourlidas (2006).

\begin{tabular}{|c|c|c|}
\hline Parameter & [units] & Description \\
\hline $2 \alpha$ & {$[\mathrm{deg}]$} & Angle between the axis of both legs \\
\hline$h$ & {$\left[r_{\text {sun }}\right]$} & Height of the legs \\
\hline$h_{\text {front }}$ & {$\left[r_{\text {sun }}\right]$} & $\begin{array}{l}\text { Distance from center of the Sun to } \\
\text { leading edge }\end{array}$ \\
\hline$\kappa$ & & Aspect ratio of $a$ and $r$ \\
\hline$\phi$ & {$[\mathrm{deg}]$} & $\begin{array}{l}\text { Carrington longitude of the projection } \\
\text { of apex on the Sun's surface }\end{array}$ \\
\hline$\theta$ & {$[\mathrm{deg}]$} & $\begin{array}{l}\text { Heliographic latitude of the projection } \\
\text { of apex on the Sun's surface }\end{array}$ \\
\hline$\gamma$ & {$[\mathrm{deg}]$} & $\begin{array}{l}\text { Tilt angle of the SR neutral line } \\
\text { relative to the solar equator }\end{array}$ \\
\hline$a$ & & $\begin{array}{l}\text { Cross section radius of the tube } \\
\text { section }\end{array}$ \\
\hline$r$ & & $\begin{array}{l}\text { Distance from center of the Sun to the } \\
\text { outer boundary of the shell }\end{array}$ \\
\hline
\end{tabular}

opposite ends of the neutral line (NL), which has a tilt angle $\gamma$ relative to the solar equator. Table 1 provides an overview of the GCS model parameters. Further information regarding the GCS Model can be found in Thernisien, Howard, and Vourlidas (2006) and Thernisien, Vourlidas, and Howard (2009).

\section{Examples of GCS Modeling of Events from the "Best-of" List}

\subsection{CME of 4 August 2009}

We apply the GCS model to the CME detected on 4 August 2009 (Figure 4) when the two STEREO spacecraft were separated by $107.5^{\circ}$ in heliographic longitude, i.e. they observed the CME from different viewing angles. The two STEREO spacecraft detected the CME at different position angles (PA) of $90^{\circ}$ and $270^{\circ}$, respectively, as shown in the COR2-A and COR2-B (left) images in Figure 4. The GCS modeling technique was applied to basedifference COR2 images after they had been processed using the standard routines (secchi_prep). ${ }^{5}$ For the fit, we selected the time when the CME was the brightest in the COR2 field of view. On 4 August 2009, the CME was modeled when it was observed at 23:22 UT when its leading edge had reached a distance of about 13 solar radii. The right panels in Figure 4 show the modeling results through overlays of the GCS wireframe flux rope geometry on the CME images. The six parameters which describe the geometry of the GCS model are summarized in Table 2.

The synthetic coronagraphic images generated with a ray-tracing code are shown in Figure 5. The ray-tracing code allows us to render the $3 \mathrm{D}$ electron density distribution into a coronagraph image taking into account Thomson scattering. In this case, the CME detected in the COR2 field of view is represented by a flux rope which has its apex located at $222^{\circ}$ in Carrington longitude lying in the solar equatorial plane (see Table 2). The radial height

\footnotetext{
${ }^{5}$ Colaninno, R.: 2006-2010, The SECCHI_PREP Homepage, Naval Research Laboratory (NRL/GMU), http://secchi.nrl.navy.mil/wiki/pmwiki.php?n=Main.HomePage.
} 


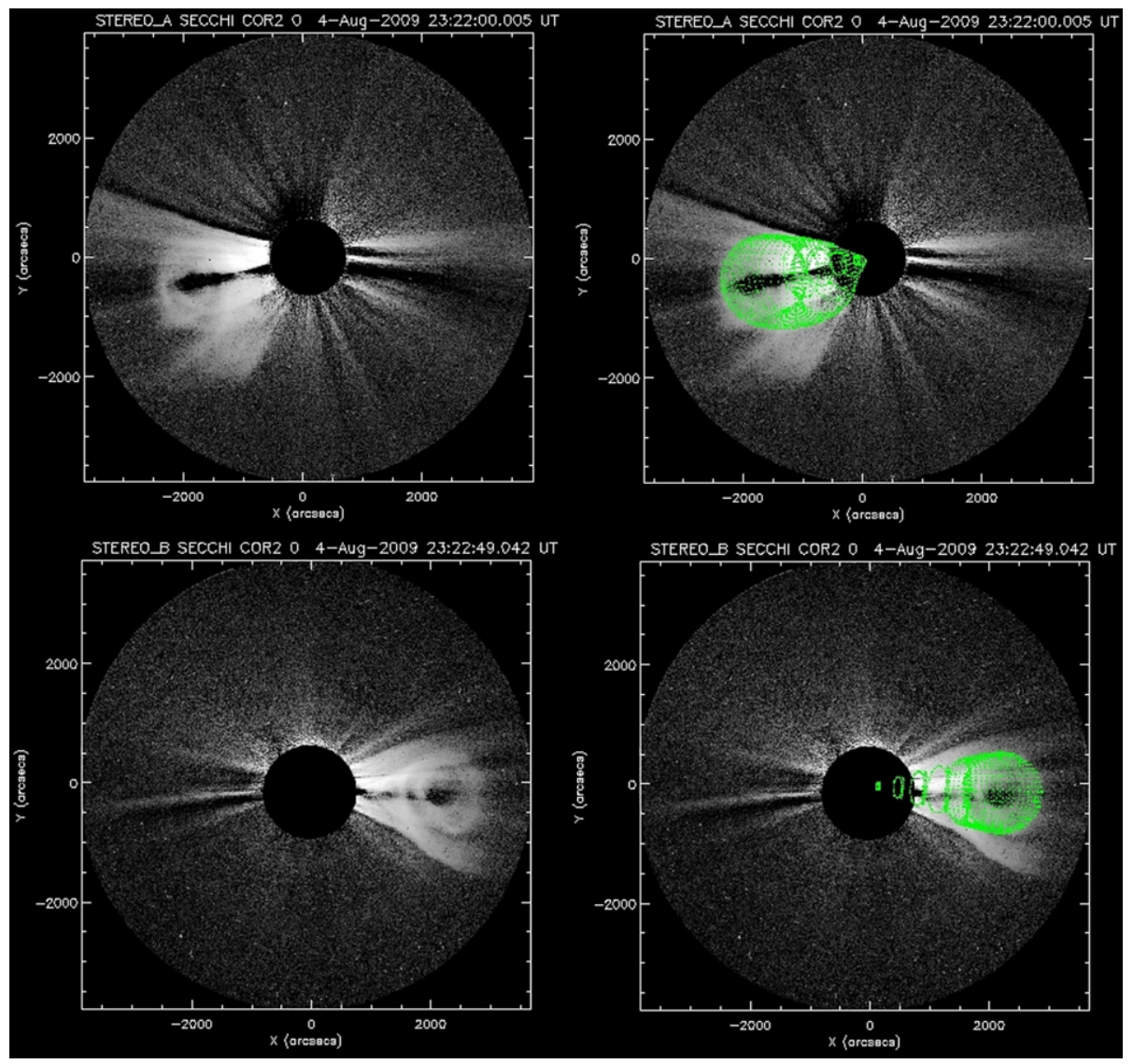

Figure 4 Left column of images: STEREO/SECCHI/COR2-A (top row) and -B (bottom row) white-light coronagraph observations of the CME detected on 4 August 2009 at 23:22 UT. The separation angle between the two spacecraft was $107^{\circ}$ in longitude. Right column of images: wireframe rendering results (overlaid in green) derived through the GCS Model.

Table 2 Model results through GCS forward-modeling of two CMEs observed on 4 August 2009 and 1 February 2010.

\begin{tabular}{llll}
\hline Parameter & & $\begin{array}{l}\text { CME 1 } \\
\text { (4 August 2009) }\end{array}$ & $\begin{array}{l}\text { CME 2 } \\
\text { (1 February 2010) }\end{array}$ \\
\hline Longitude & $\phi[\mathrm{deg}]$ & 222.5 & 39.1 \\
Latitude & $\theta[\mathrm{deg}]$ & -2.8 & -18.4 \\
Tilt angle & $\gamma[\mathrm{deg}]$ & 0.0 & 15.1 \\
Height & $h_{\mathrm{f}}\left[r_{\mathrm{S}}\right]$ & 13.4 & 16.8 \\
Aspect ratio & $\kappa$ & 0.3 & 0.3 \\
Half angle & $\alpha[\mathrm{deg}]$ & 12.0 & 23.2 \\
\hline
\end{tabular}

of its leading edge is 13 solar radii. Figure 5 further shows the modeled flux rope footpoints and apex locations projected onto the EUVI $195 \AA$ images (right column) for the time of the COR2 modeling. Green crosses signify that the flux rope is located on the visible side of the solar disk whereas white crosses define a backsided flux rope. The white-light structure of 

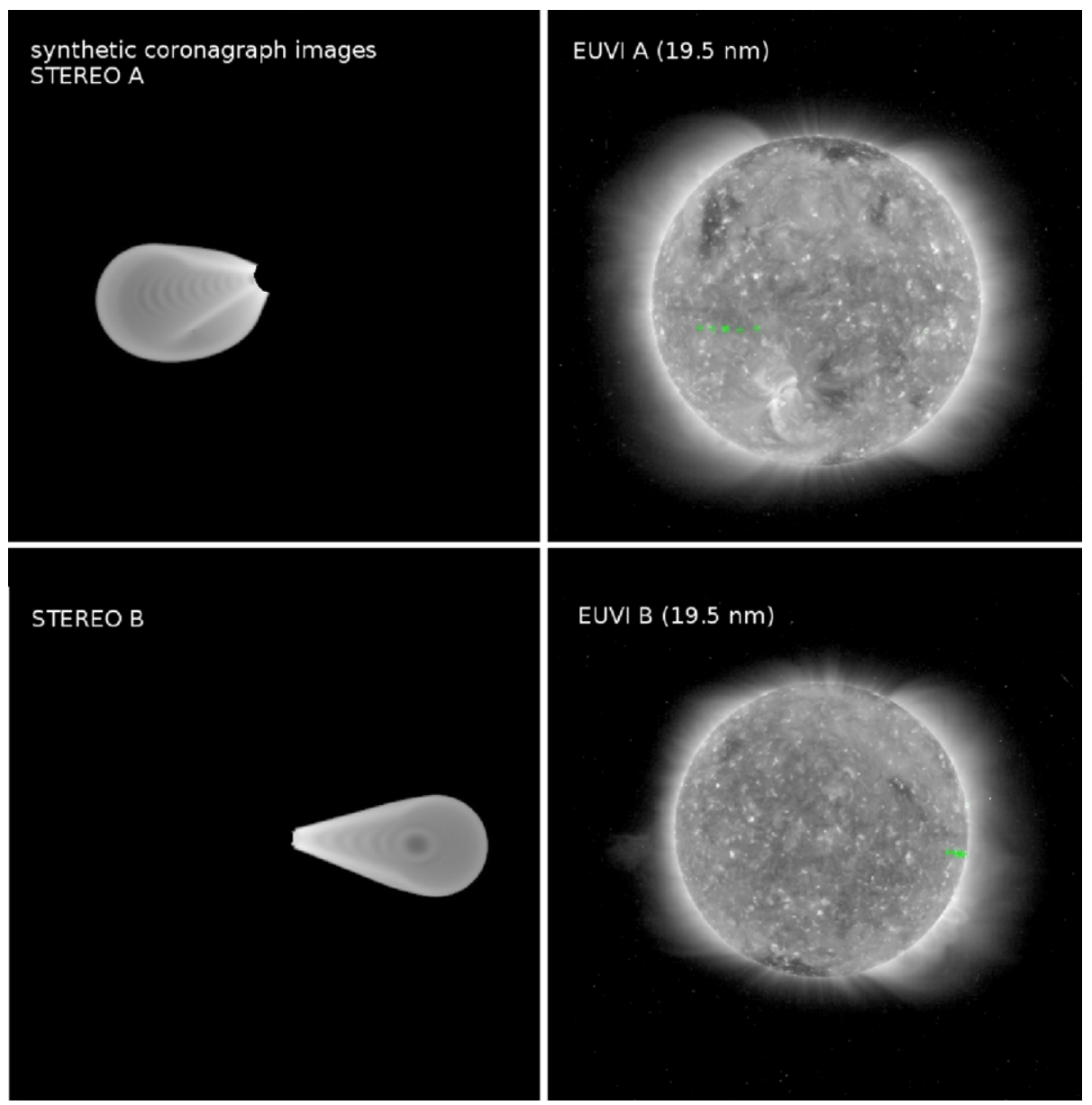

Figure 5 Left column: GCS synthetic coronagraph images for the CME observed on 4 August 2009 at 23:22 UT as shown in Figure 4. The separation angle between the two spacecraft was $107^{\circ}$ in longitude. Right column: STEREO/SECCHI/EUVI-A (top) and -B (bottom) observations at $195 \AA$ with projection of the flux rope footpoints and apex on the Sun's surface.

the 4 August 2009 CME reveals features similar to many other cases of the "COR2 Best-of CME" list.

\subsection{CME of 1 February 2010}

As discussed earlier solar activity as represented by the sunspot number and the monthly rate of CMEs has shown increased levels since about February 2010. In contrast to a CME typical of the solar minimum years, Figure 6 shows a CME detected on 1 February 2010, i.e. in the early rising phase of Cycle 24. At this time STEREO-A and -B were separated by $135.3^{\circ}$ in heliographic longitude and observed the CME at PAs of about $180^{\circ}$ and $225^{\circ}$, respectively. The GCS modeling results are summarized in Table 2. The flux rope parameters fitting this CME differ from those of the 4 August 2009 event. In this case, the flux rope apex was located $18^{\circ}$ south of the solar equator and exhibited a tilt angle of $15^{\circ}$. The 

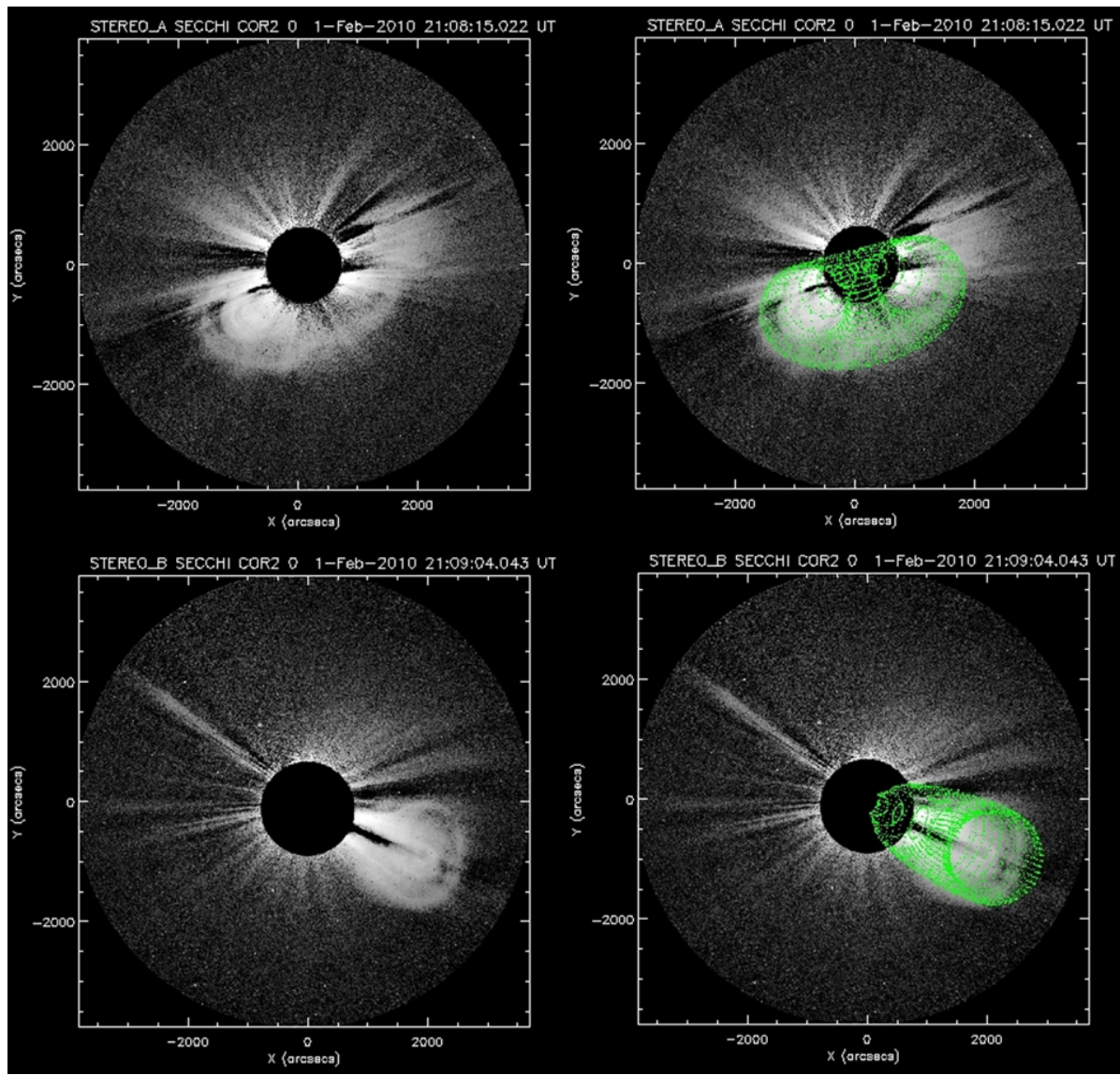

Figure 6 Left column of images: STEREO/SECCHI/COR2-A (top) and -B (bottom) white-light coronagraph observations of the CME detected on 1 February 2010 at 21:08 UT. The separation angle between the two spacecraft was $135^{\circ}$ in longitude. Right column of images: wireframe rendering results (overlaid in green) derived through the GCS Model.

angular width was $46^{\circ}$, i.e. its calculated half angle of $23^{\circ}$ was double the size of the August event. During this time period CMEs generally started looking wider and more massive in white-light coronagraph images. Because of the large angular separation of the COR2-A and -B instruments, the CME looks different in the COR2-A and -B images. The GCS synthetic coronagraphic images generated with the ray-tracing code for this event are shown in Figure 7 together with EUVI-A and -B observations taken at $195 \AA$.

\subsection{GCS Modeling Results for "COR 2 Best-of CMEs"}

Out of the 120 events of the "STEREO/SECCHI/COR2 Best-of List"' 51 events have been modeled similarly to the sample events described in Sections 3.1 and 3.2. The modeled

\footnotetext{
${ }^{6}$ The complete "Best-of" CME list is shown in Table 3 in the Appendix and in Table 3 in the Electronic Supplementary Material.
} 


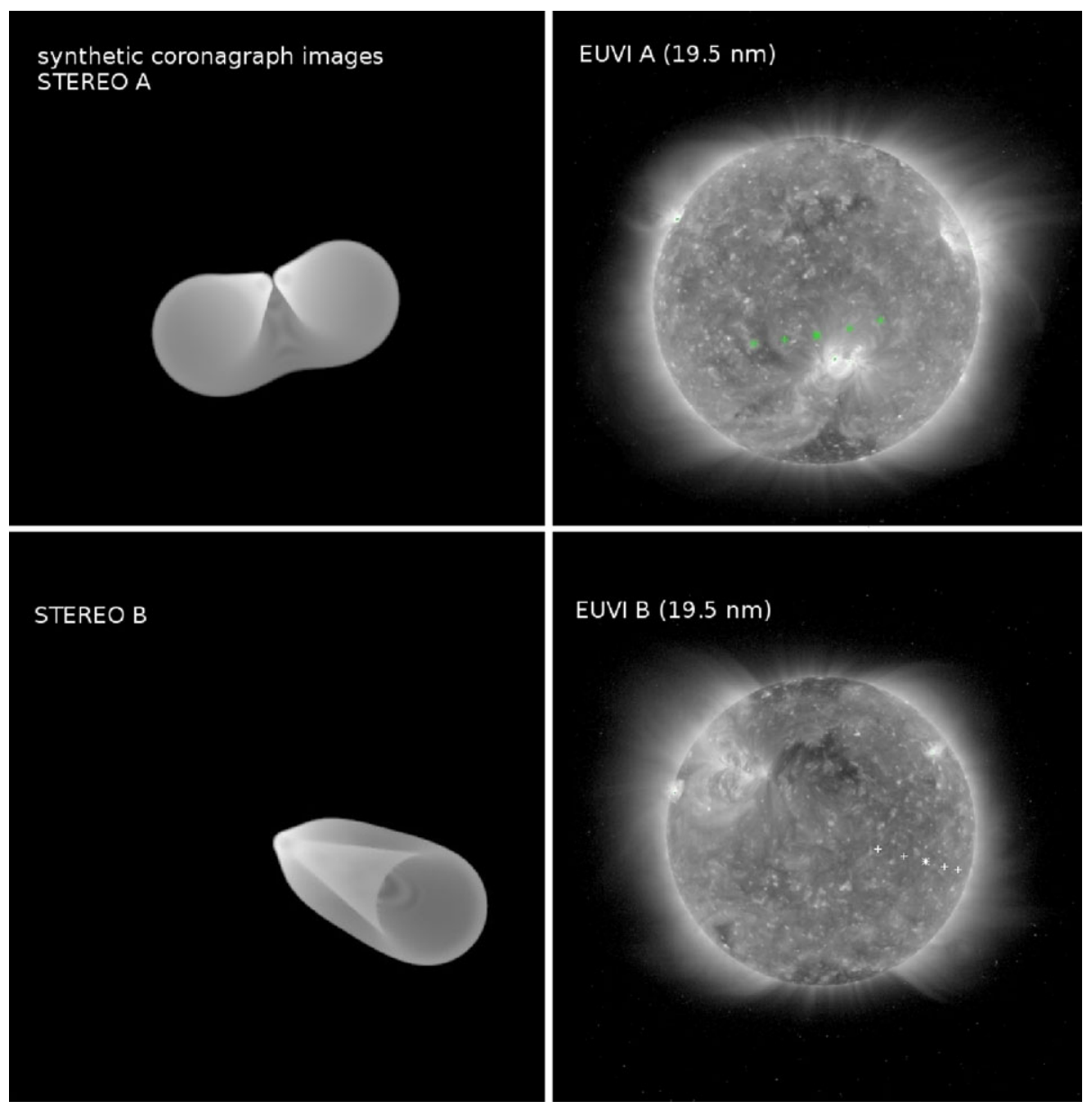

Figure 7 Left column: GCS synthetic coronagraph images for the CME observed on 1 February 2010 at 21:08 UT as shown in Figure 6. The separation angle between the two spacecraft was $135^{\circ}$ in longitude. Right column: STEREO/SECCHI/EUVI-A (top) and -B (bottom) observations at $195 \AA$ with projection of the flux rope footpoints and apex on the Sun's surface.

events hitherto were from 2010 because they appeared brighter and were easier to model than the fainter cases appearing at solar minimum. Figure 8 shows the calculated latitudes for 51 flux rope apexes resulting from the modeling of the CMEs in 2010. In 2010 the flux rope apexes were located between $30^{\circ}$ southern and $40^{\circ}$ northern latitude. Figure 9 shows the calculated GCS flux rope tilt angles plotted versus time in 2010. The tilt angle of a modeled flux rope denotes the angle between the line between its footpoints and apex which are projected on the solar surface and the solar equator. The flux rope is oriented parallel to the equator for an angle of $0^{\circ}$ and perpendicular for $90^{\circ}$. The CMEs observed north and south of the solar equator show a similar pattern of scatter in the range of up to $30-40^{\circ}$. It is interesting to note that apart from one exception, flux ropes with a tilt angle larger than roughly $40^{\circ}$ are lacking. In this context it is important for further studies to inspect the tilt angle of the remaining "Best-of CMEs" observed between 2007 and 2009, because Thernisien, Vourlidas, and Howard (2009) found that e.g. the CMEs on 31 December 2007 
Figure 8 Distribution of latitude of the calculated apex position projected on the solar surface for 51 GCS-modeled CMEs observed in 2010.

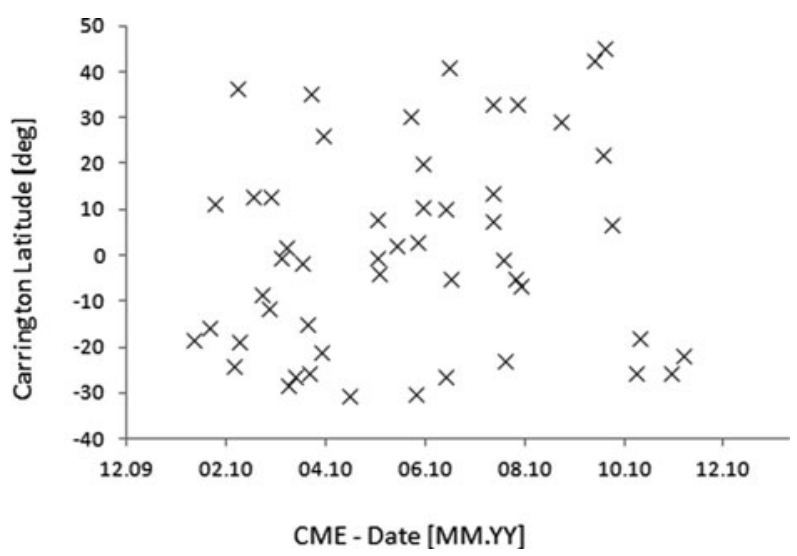

Figure 9 Tilt angle distribution of the line of modeled footpoints and apex projected on the solar surface for 51 GCS-modeled CMEs observed in 2010.

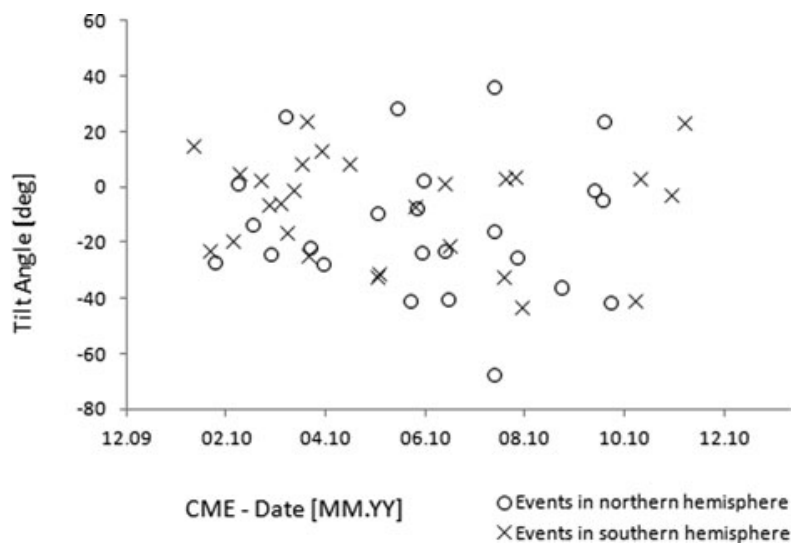

and 23 January 2008 exhibit a large tilt angle of $90^{\circ}$ and $-49^{\circ}$, respectively. A further investigation of this aspect is needed to understand better the inclination characteristics of flux rope CMEs. Figure 10 shows the distribution of the GCS flux rope half angles $\alpha$ which represents through $2 \alpha$ the separation angle between both legs of the flux rope. The typical half angle of the flux ropes is estimated to lie between $10^{\circ}$ and $25^{\circ}$ during 2010 for a CME observed between 10 and 15 solar radii. A half angle of $10^{\circ}$ to $25^{\circ}$ corresponds to an angular width of the $\mathrm{CME}$ lying between $20^{\circ}$ and $50^{\circ}$ which is comparable to the typical angular width of CMEs observed by SOHO/LASCO (e.g. St. Cyr et al., 2000; Yashiro et al., 2004).

It should be noted that since the fits are done by hand they exhibit the modeler's subjective understanding of the observed CME. Hence the fit results depend to a certain extent on the experience of the user for the interpretation of the CME white-light observation. In this context Thernisien, Vourlidas, and Howard (2009) used a merit function to determine how well the model is able to reproduce an observed CME's white-light structure. After performing a sensitivity analysis of the model parameters, the authors found that the deviations in the parameters $\gamma$ and $\alpha$ are an order of magnitude larger than the deviations in the longitude and latitude. Hence the values for the tilt angle may exhibit a larger uncertainty than the one of the other parameters. 
Figure 10 Distribution of the calculated flux rope half angle for 51 GCS-modeled "Best-of CMEs" observed in 2010.

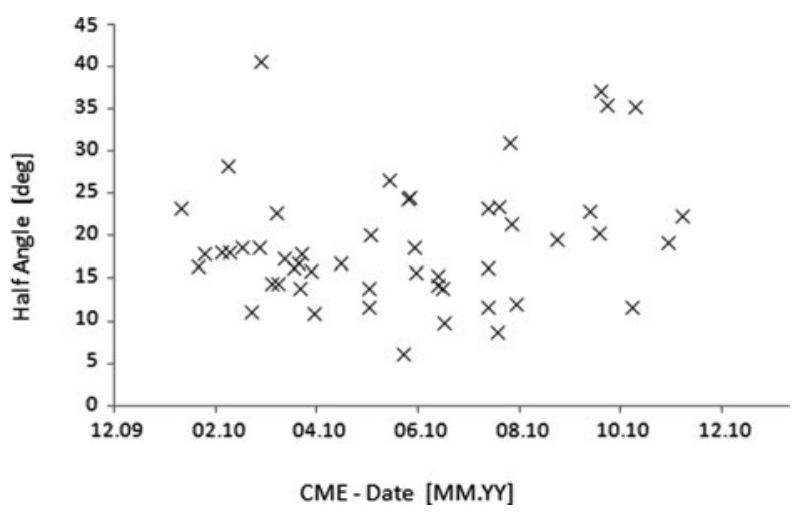

\section{Comparison with source region and discussion}

To compare the calculated GCS parameters (Table 4) of the flux ropes with the CME associated source region characteristics, we investigated the source region for each CME using SECCHI and SOHO/MDI data. For each modeled CME event we used the SECCHI/COR1 observations to track the CME back towards the low corona and then used the EUVI $195 \AA$ and $304 \AA$ observations to identify the coronal SR.

After identification of a CME's source region we compared the calculated apex position provided by the GCS modeling to the SR location. Figure 11 shows a histogram of the differences between the SR longitude and the modeled apex position in bins of $15^{\circ}$ Carrington longitude for 39 events. For the 12 remaining CMEs no associated SRs could be determined. We find that for $82 \%$ (32 out of 39) of the CME events the discrepancy is not larger than $30^{\circ}$. Larger deviations occur only for a small number of events (7 out of 39) and the larger differences are decreasingly frequent. A similar behavior is found for the difference in solar latitude between identified SR and modeled apex position as shown in Figure 12. Here $82 \%$ (32 out of 39) of all CME events exhibit a discrepancy of less than $25^{\circ}$ in solar latitude. Considering a $10^{\circ}$ difference as insignificant, we can conclude that $41 \%$ of all CMEs do not deflect latitudinally while the rest of the same exhibits a very modest $23^{\circ}$ average deflection to lower latitudes.

Next we projected the calculated apexes onto SOHO/MDI (Michelson Doppler Imager) Synoptic Charts ${ }^{7}$ shown for the CME event observed on 4 June 2010. The center of the observed SR is labeled with a white plus sign and is located within a magnetic bipolar region. For a better visibility the SR is surrounded by a white circle. The radius of the circle is arbitrary with no reference to the spatial extent of the SR. In this case we assume a prominence as the SR, indicated with "P". The position of the apex is marked with a green asterisk and the footpoints with green squares connected with a line which simultaneously denotes the orientation of the flux rope axis. The length of the footpoint line corresponds to the half angle $\alpha$, respectively, $2 \alpha$, the angle between both legs of the flux rope. In this case the apex projection lies only $13^{\circ}$ south of the identified CME SR with an offset of only $16^{\circ}$ in solar longitude. The MDI map reveals a bipolar photospheric region as a source of the analyzed CME as found in the studies of Tripathi, Bothmer, and Cremades (2004). In

\footnotetext{
${ }^{7}$ Stanford-Lockheed Institute for Space Research, W.W. Hansen Experimental Physics Laboratory (HEPL), Stanford University: 2010, MDI Magnetic Field and Intensity Synoptic Charts, http://soi.stanford.edu/ magnetic/index6.html.
} 
Figure 11 Differences in Carrington Longitude between observed SR and GCS-modeled apex position in bins of $15^{\circ}$ for $39 \mathrm{CME}$ events and their associated source regions. For the 12 remaining CMEs no associated SRs could be determined.
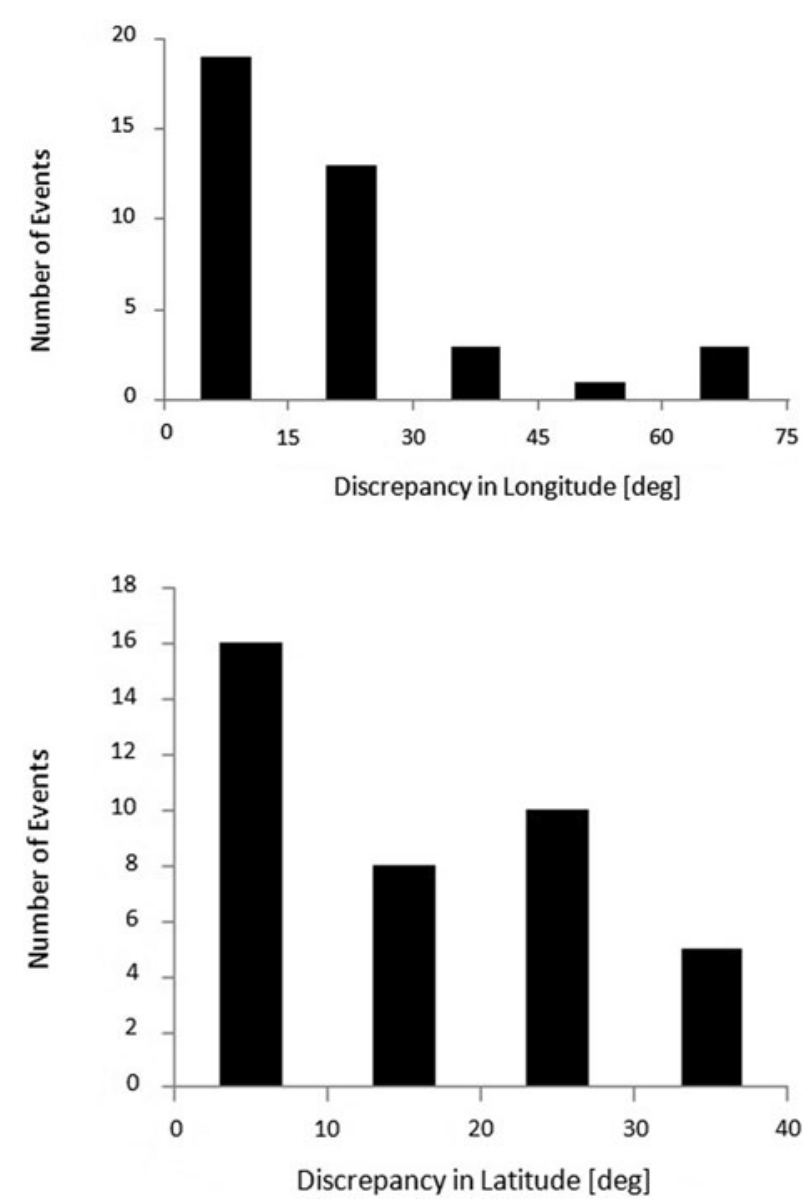

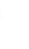

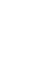

Figure 12 Differences in latitude between observed SR and GCS-modeled apex position in bins of $10^{\circ}$ for $39 \mathrm{CME}$ events and their associated source regions. For the 12 remaining CMEs no associated SRs could be determined. 


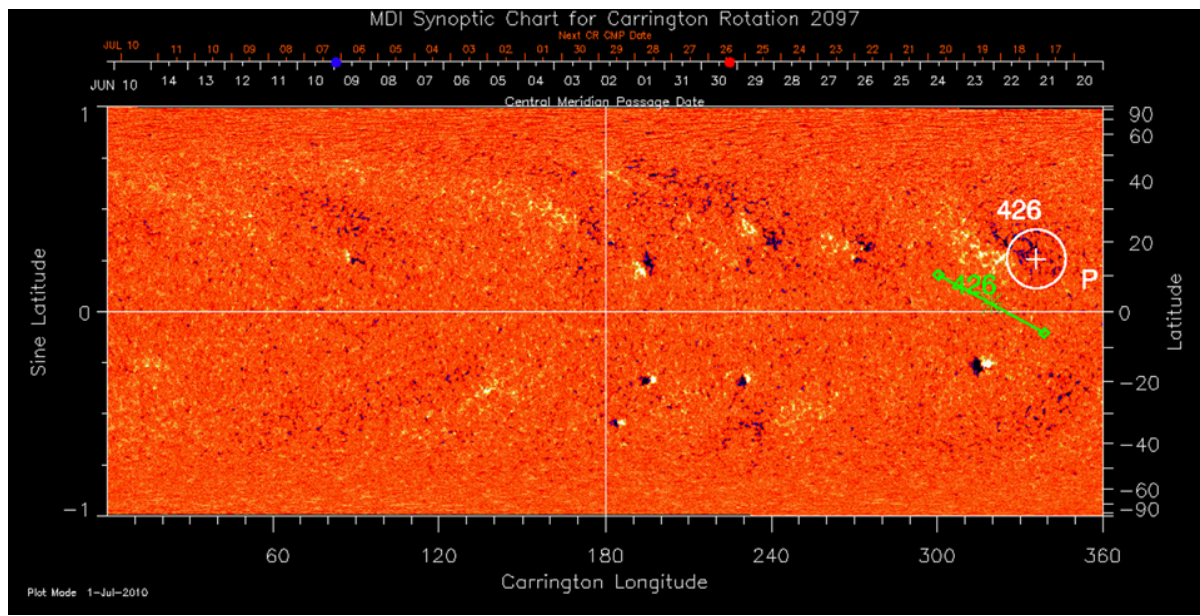

Figure 13 SOHO/MDI Synoptic Chart for Carrington Rotation 2097 labeled with the center of observed SR (white encircled plus sign) and the position of apex and footpoints of the GCS modeled CME observed on 4 June 2010 (green).

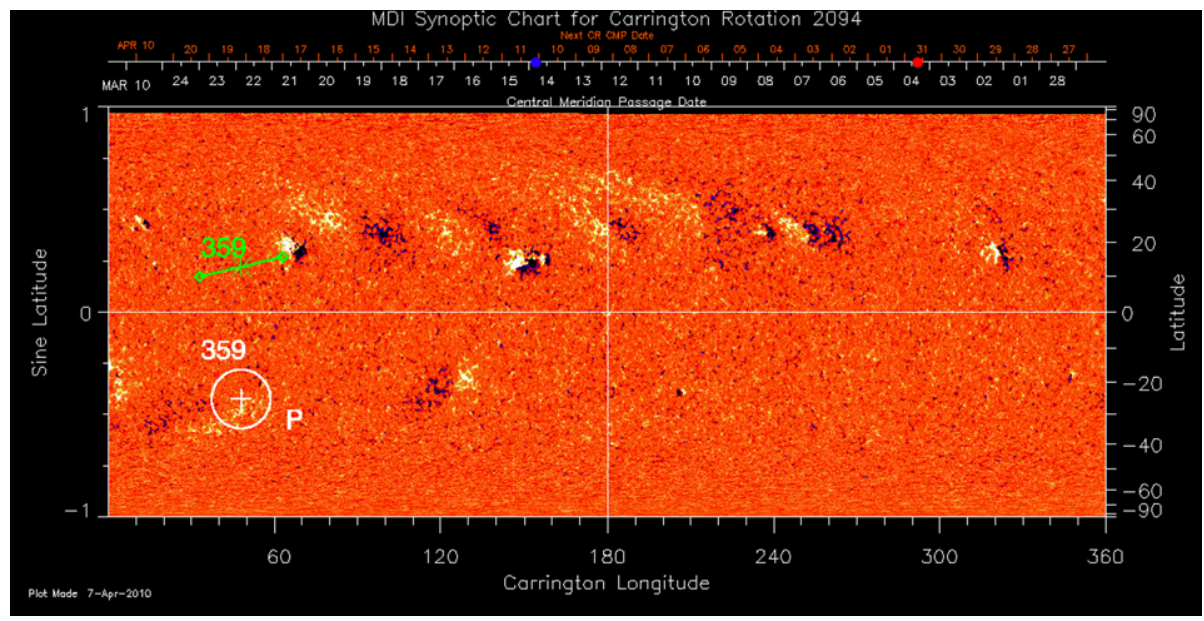

Figure 14 SOHO/MDI Synoptic Chart for Carrington Rotation 2094 labeled with the center of observed SR (white) and the position of apex and footpoints of the GCS modeled CME observed on 8 March 2010 (green).

rise by a factor of 3 to 4 between September 2009 and March 2010. This increase can be interpreted as the start of the overall rise of solar activity towards the next solar maximum expected around the year 2012-2013.

From the SOTERIA COR2 CME list we selected 120 events as a "Best-of" list based on their brightness appearance in the COR2 field of view. Fifty-one of the "Best-of CMEs" have been modeled using the GCS forward-modeling technique developed by Thernisien, Howard, and Vourlidas (2006) and Thernisien, Vourlidas, and Howard (2009) to infer the CME's 3D structure. The modeling results reveal: 
- A good fit of the observed CME white-light structure as GCS flux ropes.

- The calculated GCS apex latitude position is between $30^{\circ}$ southern and $40^{\circ}$ northern hemisphere of the solar equator for CMEs observed in 2010.

- The tilt angle for GCS modeled flux ropes is distributed between roughly $\pm 40^{\circ}$.

- The flux rope half angle extends from $10^{\circ}$ up to $25^{\circ}$ which corresponds to an angular width of the CME lying between $20^{\circ}$ and $50^{\circ}$.

From the comparison of the GCS modeled apex position with the identified associated source region position it is found that in $82 \%$ of the CME events the discrepancy extends from $0^{\circ}$ up to $30^{\circ}$ in Carrington longitude. Larger deviations occurred only for a smaller number of events and the larger differences are also less frequent. A similar behavior is found for the difference in solar latitude between the identified SR and modeled apex positions. Here $82 \%$ of all CME events exhibit a discrepancy of less than $25^{\circ}$ in solar latitude. These findings imply that the observed CMEs were commonly deflected away from the radial direction over the first few solar radii.

Some issues which were not discussed in detail in this study but are important and very interesting pertain to error bars of the GCS model parameters. So for example, it should be considered that the deviation for the parameters $\gamma$ and $\alpha$ are an order of magnitude larger than the deviation for the longitude and latitude. Hence the values for the tilt angle exhibit a larger uncertainty than those for the other parameters (Thernisien, Vourlidas, and Howard, 2009). Further analysis of the calculated GCS flux rope parameters and the investigation of possible $\mathrm{CME}$ deflection and $\mathrm{CME}$ distortion will be undertaken in the near future.

Acknowledgements The research leading to these results has received funding from the European Community's Seventh Framework Programme (FP7/2007-2013) under the grant agreement $n^{\circ} 218816$ (SOTERIA project, www.soteria.eu). Volker Bothmer acknowledges support of the project Stereo/Corona by the German Bundesministerium für Bildung und Forschung through the deutsche Zentrum für Luft-und Raumfahrt e.V. (DLR, German Space 59 Agency) as a collaborative effort with the Max-Planck-Institut für Sonnensystemforschung (MPS) under grant $50{ }^{\circ} \mathrm{C} 0904$. Stereo/Corona is a science and hardware contribution to the optical image package SECCHI, developed for the NASA STEREO mission. The STEREO/SECCHI data used for this study are prepared by an international consortium of NASA Goddard Space Flight Center (USA), Lockheed Martin Solar and Astrophysics Lab (USA), Naval Research Laboratory (USA), Rutherford Appleton Laboratory (UK), University of Birmingham (UK), Max-Planck-Institut für Sonnensystemforschung (Germany), Institut d'Optique Thèorique et Appliquèe (France), Institut d'Astrophysique Spatiale (France) and Centre Spatiale de Liège (Belgium). The NRL effort was supported by NASA, the USAF Space Test Program and the Office of Naval Research. The work of Angelos Vourlidas and Russell A. Howard is supported by NASA contract S-136361-Y to the Naval Research Laboratory. Data from the SOHO/MDI instrument are courtesy of the SOHO/MDI consortium. The SOHO/MDI data are produced by an international consortium of the Naval Research Laboratory (USA), Max-Planck-Institut für Aeronomie (Germany), Laboratoire d'Astronomie (France) and the University of Birmingham (UK). SOHO is an international project of collaboration between ESA and NASA.

Open Access This article is distributed under the terms of the Creative Commons Attribution License which permits any use, distribution, and reproduction in any medium, provided the original author(s) and the source are credited. 


\section{Appendix}

\section{“Best-of CME” List:}

Table 3 "Best-of CME" list with 120 CMEs detected with STEREO/SECCHI/COR2- A/B for the time period from January 2007 until December 2010. No indicates the CME-Number in the COR2 list, modeled CMEs are marked with $m$. Date and time ( $n$ stands for next day) corresponds to the observation time with COR2-A and -B, respectively. $P A$ denotes the position angle corresponding to the COR2 field of view with $0^{\circ}$ pointing to the solar north and increasing angle counter-clockwise. $A$ and $B$ in the last column indicate how good the CME was visible in COR2-A and -B observations for the user (- - very faint, - faint, + good and ++ very good). $H$ stands for Halo and $p H$ for partial Halo CME.

\begin{tabular}{|c|c|c|c|c|c|c|}
\hline No & $\begin{array}{l}\text { Date } \\
\text { yyyymmdd }\end{array}$ & $\begin{array}{l}\text { Time(A) } \\
\text { hh:mm:ss }\end{array}$ & $\begin{array}{l}\mathrm{PA}(\mathrm{A}) \\
{[\mathrm{deg}]}\end{array}$ & $\begin{array}{l}\text { Time (B) } \\
\text { hh:mm:ss }\end{array}$ & $\begin{array}{l}\text { PA(B) } \\
\text { [deg] }\end{array}$ & Comments \\
\hline 005 & 20070124 & $15: 02: 40$ & 90 & $15: 02: 46$ & 90 & $\mathrm{~A}++, \mathrm{B}-$ \\
\hline 007 & 20070130 & 11:02:40 & 270 & $12: 02: 48$ & 270 & $\mathrm{~A}+, \mathrm{B}-$ \\
\hline 020 & 20070312 & 00:02:40 & 90 & 00:03:01 & 90 & $\mathrm{~B}-, \mathrm{A}+$ \\
\hline 025 & 20070331 & $09: 52: 30$ & 270 & $09: 52: 58$ & 270 & $\mathrm{~B}+$ \\
\hline 035 & 20070509 & $04: 22: 30$ & 90 & $04: 53: 14$ & 90 & $\mathrm{~A}+, \mathrm{B}-$ \\
\hline 038 & 20070515 & $19: 22: 30$ & 45 & $20: 23: 17$ & 45 & $\mathrm{~A}++, \mathrm{B}-$ \\
\hline 049 & 20070604 & $21: 22: 30$ & 270 & $03: 23: 24 n$ & 270 & $\mathrm{~A}+, \mathrm{B}-$ \\
\hline 050 & 20070607 & $20: 22: 30$ & 225 & $21: 23: 25$ & 225 & $\mathrm{~A}+, \mathrm{B}-$ \\
\hline 060 & 20070708 & $20: 22: 30$ & 90 & $21: 23: 32$ & 90 & $\mathrm{~A}+, \mathrm{B}-$ \\
\hline 071 & 20070821 & $08: 52: 30$ & 270 & $10: 53: 33$ & 270 & $\mathrm{~A}+, \mathrm{B}-$ \\
\hline 078 & 20071008 & $17: 22: 30$ & 270 & $16: 53: 22$ & 270 & $\mathrm{~A}-, \mathrm{B}-$ \\
\hline 083 & 20071104 & $13: 52: 20$ & 45 & 20:23:02 & 45 & $\mathrm{~A}+, \mathrm{B}--$ \\
\hline 086 & 20071116 & $10: 52: 20$ & 225 & $12: 52: 57$ & 225 & $\mathrm{~A}+, \mathrm{B}--$ \\
\hline 092 & 20071231 & 01:22:20 & 135 & 01:52:42 & 135 & $\mathrm{~A}++, \mathrm{B}+$ \\
\hline 093 & 20080102 & $10: 22: 20$ & 90 & $11: 22: 41$ & 90 & $\mathrm{~A}++, \mathrm{B}+$ \\
\hline 095 & 20080122 & $23: 52: 20$ & 180 & $23: 52: 38$ & 135 & $\mathrm{~A}+, \mathrm{B}+$ \\
\hline 096 & 20080129 & $00: 22: 20$ & 270 & $00: 22: 37$ & 270 & $\mathrm{~A}+, \mathrm{B}-$ \\
\hline 100 & 20080212 & $07: 52: 20$ & 225 & $09: 22: 36$ & 225 & $\mathrm{~A}+, \mathrm{B}-$ \\
\hline 104 & 20080223 & $20: 52: 20$ & 45 & $17: 52: 37$ & 45 & $\mathrm{~A}+, \mathrm{B}-$ \\
\hline 105 & 20080227 & $12: 52: 20$ & 270 & $10: 22: 37$ & 270 & $\mathrm{~A}+, \mathrm{B}-$ \\
\hline 107 & 20080317 & $15: 52: 20$ & 270 & $12: 52: 40$ & 270 & $\mathrm{~B}-, \mathrm{A}--$ \\
\hline 111 & 20080325 & $19: 22: 20$ & 90 & $19: 52: 42$ & 90 & $\mathrm{~A}+, \mathrm{B}-$ \\
\hline 113 & 20080405 & $16: 22: 20$ & 270 & $16: 52: 45$ & 270 & $\mathrm{~A}+, \mathrm{B}-$ \\
\hline 121 & 20080426 & $14: 52: 20$ & 45 & $15: 52: 53$ & 315 & $\mathrm{~A}+, \mathrm{B}--$ \\
\hline 125 & 20080517 & $10: 52: 20$ & 90 & $12: 23: 01$ & 90 & $\mathrm{~A}+, \mathrm{B}--$ \\
\hline 130 & 20080601 & $23: 22: 20$ & 90 & - & - & $\mathrm{A}+$ \\
\hline 141 & 20080731 & $06: 22: 20$ & 270 & - & - & $\mathrm{A}-$ \\
\hline 145 & 20080908 & $02: 22: 20$ & 270 & $03: 53: 22$ & 90 & $\mathrm{~A}--, \mathrm{B}+$ \\
\hline 156 & 20081017 & $09: 52: 20$ & 270 & $10: 53: 14$ & 90 & $\mathrm{~A}-, \mathrm{B}+$ \\
\hline 158 & 20081026 & $23: 52: 20$ & 270 & $22: 23: 11$ & 270 & $\mathrm{~A}--, \mathrm{B}+$ \\
\hline 162 & 20081103 & 01:22:20 & 45 & 01:53:09 & 315 & $\mathrm{~A}--, \mathrm{B}-$ \\
\hline 167 & 20081113 & $16: 22: 20$ & 225 & $15: 23: 06$ & 135 & $\mathrm{~A}+, \mathrm{B}-$ \\
\hline 172 & 20081123 & $22: 22: 20$ & 270 & $04: 23: 02 n$ & 270 & $\mathrm{~A}+, \mathrm{B}-$ \\
\hline 175 & 20081208 & $17: 22: 20$ & 315 & - & - & $\mathrm{A}++$ \\
\hline
\end{tabular}


Table 3 (Continued)

\begin{tabular}{|c|c|c|c|c|c|c|}
\hline No & $\begin{array}{l}\text { Date } \\
\text { yyyymmdd }\end{array}$ & $\begin{array}{l}\text { Time(A) } \\
\text { hh:mm:ss }\end{array}$ & $\begin{array}{l}\mathrm{PA}(\mathrm{A}) \\
{[\mathrm{deg}]}\end{array}$ & $\begin{array}{l}\text { Time (B) } \\
\text { hh:mm:ss }\end{array}$ & $\begin{array}{l}\mathrm{PA}(\mathrm{B}) \\
{[\mathrm{deg}]}\end{array}$ & Comments \\
\hline 176 & 20081212 & $08: 22: 20$ & 90 & $08: 22: 55$ & 270 & $\mathrm{~A}-, \mathrm{B}+$ \\
\hline 179 & 20081227 & $06: 52: 20$ & 45 & $10: 52: 50$ & 315 & $\mathrm{~A}-, \mathrm{B}--$ \\
\hline 186 & 20090114 & $06: 22: 00$ & 90 & $11: 22: 00$ & 270 & $\mathrm{~A}+, \mathrm{B}--$ \\
\hline 188 & 20090117 & - & - & $14: 22: 24$ & 90 & $\mathrm{~B}+$ \\
\hline 195 & 20090210 & $20: 52: 00$ & 270,90 & $01: 52: 19 n$ & 225,90 & $\mathrm{~A}+, \mathrm{B}--$ \\
\hline 198 & 20090218 & 09:22:00 & 90 & $15: 22: 19$ & $45-315$ & $\mathrm{~A}+, \mathrm{B}--$ \\
\hline 207 & 20090316 & $14: 22: 00$ & 90,270 & $12: 22: 19$ & 90,270 & $\mathrm{~A}--, \mathrm{B}+$ \\
\hline 208 & 20090318 & $00: 22: 00$ & 90 & $07: 22: 19$ & 315 & $\mathrm{~A}+, \mathrm{B}--$ \\
\hline 213 & 20090326 & 09:22:00 & 270 & $19: 22: 20$ & 135 & $\mathrm{~A}+, \mathrm{B}+$ \\
\hline 217 & 20090410 & $19: 22: 00$ & 270 & - & - & $\mathrm{A}+$ \\
\hline 226 & 20090423 & $00: 22: 00$ & 90,315 & $00: 22: 26$ & 90 & $\mathrm{~A}+, \mathrm{B}-$ \\
\hline 228 & 20090502 & $23: 22: 00$ & 270 & $20: 22: 28$ & 90 & $\mathrm{~A}--, \mathrm{B}+$ \\
\hline 234 & 20090521 & - & - & $19: 22: 35$ & 135 & $\mathrm{~B}+$ \\
\hline 243 & 20090613 & $13: 52: 00$ & 90 & $14: 52: 43$ & 270 & $\mathrm{~A}+, \mathrm{B}-$ \\
\hline 244 & 20090615 & $02: 52: 00 n$ & 90 & $21: 22: 43$ & 270 & $\mathrm{~A}+, \mathrm{B}-$ \\
\hline 245 & 20090616 & $16: 52: 00$ & 270 & $16: 52: 44$ & 270 & $\mathrm{~A}+, \mathrm{B}-$ \\
\hline 259 & 20090723 & $08: 22: 00$ & 270 & $06: 52: 49$ & 90 & $\mathrm{~A}-, \mathrm{B}+$ \\
\hline 262 & 20090804 & $18: 22: 00$ & 90 & $18: 22: 49$ & 270 & $\mathrm{~A}+, \mathrm{B}+$ \\
\hline 263 & 20090808 & $16: 52: 00$ & 90 & $22: 22: 49$ & 90 & $\mathrm{~A}+, \mathrm{B}--$ \\
\hline 264 & 20090811 & $14: 22: 49$ & 90 & $20: 22: 00$ & 270 & $\mathrm{~A}-, \mathrm{B}++$ \\
\hline 265 & 20090813 & 03:22:00 & 270 & - & - & $\mathrm{A}+$ \\
\hline 267 & 20090819 & 03:08:15 & 270 & 05:09:04 & 90 & $\mathrm{~A}+, \mathrm{B}-$ \\
\hline 271 & 20090903 & $12: 08: 15$ & 90 & 05:09:04 & 270 & $\mathrm{~A}-, \mathrm{B}+$ \\
\hline 288 & 20091031 & 05:08:15 & 90 & 05:09:04 & 270 & $\mathrm{~A}-, \mathrm{B}+$ \\
\hline 291 & 20091108 & $05: 08: 15$ & 270 & 06:09:04 & 45 & $\mathrm{~A}++, \mathrm{B}-$ \\
\hline 293 & 20091115 & 13:08:15 & 270 & - & - & $\mathrm{A}-$ \\
\hline 299 & 20091121 & 08:08:15 & 90 & 11:09:04 & 270 & $\mathrm{~A}++, \mathrm{B}--$ \\
\hline 307 & 20091216 & 03:08:15 & 90 & 04:08:58 & 270 & $\mathrm{~A}+, \mathrm{B}-$ \\
\hline $325 \mathrm{~m}$ & 20100201 & $23: 08: 15$ & $135-270$ & 22:09:04 & 225 & $\mathrm{~A}--, \mathrm{B}++$ \\
\hline $333 \mathrm{~m}$ & 20100211 & $21: 08: 15$ & 90 & 23:08:41 & 270 & $\mathrm{~A}-, \mathrm{B}++$ \\
\hline $337 \mathrm{~m}$ & 20100214 & 08:08:15 & 45 & 05:08:40 & 270 & $\mathrm{~A}-, \mathrm{B}+$ \\
\hline $347 \mathrm{~m}$ & 20100225 & 01:08:15 & 135,225 & 02:08:38 & 225,45 & $\mathrm{~A}++, \mathrm{B}-$ \\
\hline $350 \mathrm{~m}$ & 20100228 & 18:08:15 & 315 & 19:08:37 & 45 & $\mathrm{~A}++, \mathrm{B}+$ \\
\hline $351 \mathrm{~m}$ & 20100301 & 09:08:15 & $135-180$ & 08:08:37 & 225 & $\mathrm{~A}-, \mathrm{B}+$ \\
\hline $359 \mathrm{~m}$ & 20100309 & 08:08:15 & 270 & $12: 08: 36$ & 90 & $\mathrm{~A}+, \mathrm{B}-$ \\
\hline $365 \mathrm{~m}$ & 20100314 & $13: 08: 15$ & 225 & $14: 08: 36$ & 90 & $\mathrm{~A}-, \mathrm{B}++$ \\
\hline $369 m$ & 20100319 & $20: 08: 15$ & 90 & 21:08:35 & 270 & $\mathrm{~A}+, \mathrm{B}++$ \\
\hline $370 \mathrm{~m}$ & 20100320 & 09:08:15 & 270 & 11:08:35 & $315-135$ & $\mathrm{~A}++, \mathrm{B}-$ \\
\hline $373 m$ & 20100326 & $16: 08: 15$ & 90 & $17: 08: 35$ & 270 & $\mathrm{~A}-, \mathrm{B}+$ \\
\hline $376 \mathrm{~m}$ & 20100329 & $12: 08: 15$ & 270 & $14: 08: 35$ & 90 & $\mathrm{~A}+, \mathrm{B}-$ \\
\hline $377 \mathrm{~m}$ & 20100330 & $12: 08: 15$ & 135,315 & $14: 08: 35$ & 45,225 & $\mathrm{~A}++, \mathrm{B}+$ \\
\hline $379 m$ & 20100403 & 11:08:15 & 135 & $12: 08: 35$ & 225 & $\mathrm{~A}++, \mathrm{B}++$ \\
\hline $383 \mathrm{~m}$ & 20100408 & 09:08:15 & 90 & $07: 08: 36$ & 270 & $\mathrm{~A}+, \mathrm{B}-$ \\
\hline $386 \mathrm{~m}$ & 20100411 & 09:08:15 & 225 & $11: 08: 36$ & 135 & $\mathrm{~A}+, \mathrm{B}-$ \\
\hline
\end{tabular}


Table 3 (Continued)

\begin{tabular}{|c|c|c|c|c|c|c|}
\hline No & $\begin{array}{l}\text { Date } \\
\text { yyyymmdd }\end{array}$ & $\begin{array}{l}\text { Time(A) } \\
\text { hh:mm:ss }\end{array}$ & $\begin{array}{l}\mathrm{PA}(\mathrm{A}) \\
{[\mathrm{deg}]}\end{array}$ & $\begin{array}{l}\text { Time (B) } \\
\text { hh:mm:ss }\end{array}$ & $\begin{array}{l}\mathrm{PA}(\mathrm{B}) \\
{[\mathrm{deg}]}\end{array}$ & Comments \\
\hline $387 \mathrm{~m}$ & 20100412 & 10:08:15 & 225 & $13: 08: 36$ & 135 & $\mathrm{~A}-, \mathrm{B}--$ \\
\hline $389 m$ & 20100413 & $15: 08: 15$ & 45 & $14: 08: 36$ & 315 & $\mathrm{~A}-, \mathrm{B}++$ \\
\hline $391 \mathrm{~m}$ & 20100419 & 05:08:15 & 135 & 04:08:36 & 225 & $\mathrm{~A}-, \mathrm{B}+$ \\
\hline $392 \mathrm{~m}$ & 20100419 & $23: 08: 15$ & 45 & $00: 08: 37 \mathrm{n}$ & 315 & $\mathrm{~A}-, \mathrm{B}++$ \\
\hline $404 \mathrm{~m}$ & 20100506 & 01:08:15 & 180 & 02:08:39 & 225 & $\mathrm{~A}-, \mathrm{B}+$ \\
\hline $415 \mathrm{~m}$ & 20100523 & 00:08:15 & 270 & 00:08:43 & 90 & $\mathrm{~A}+, \mathrm{B}-$ \\
\hline $416 \mathrm{~m}$ & 20100523 & 21:08:15 & 90 & $22: 08: 43$ & 270 & $\mathrm{~A}+, \mathrm{B}++$ \\
\hline $418 \mathrm{~m}$ & 20100524 & 17:08:15 & 90 & $17: 08: 43$ & 270 & $\mathrm{~A}-, \mathrm{B}+$ \\
\hline $426 \mathrm{~m}$ & 20100604 & $16: 08: 15$ & 270 & $16: 08: 46$ & 90 & $\mathrm{~A}+, \mathrm{B}++$ \\
\hline $429 m$ & 20100612 & 16:08:15 & 45 & 18:08:49 & 0 & $\mathrm{~A}+, \mathrm{B}-$ \\
\hline $432 \mathrm{~m}$ & 20100615 & 03:08:15 & 225,45 & 04:08:49 & 135,0 & $\mathrm{~A}+, \mathrm{B}+$ \\
\hline $434 \mathrm{~m}$ & 20100616 & 19:08:15 & 90 & 20:08:50 & 270 & $\mathrm{~A}+, \mathrm{B}-$ \\
\hline $435 \mathrm{~m}$ & 20100619 & 03:08:15 & 315 & 04:08:51 & 45 & $\mathrm{~A}+, \mathrm{B}--$ \\
\hline $437 \mathrm{~m}$ & 20100620 & $22: 08: 15$ & 90 & $23: 08: 15$ & 270 & $\mathrm{~A}-, \mathrm{B}-$ \\
\hline $444 m$ & 20100703 & 07:08:15 & 270 & 08:08:55 & 90 & $\mathrm{~A}+, \mathrm{B}++$ \\
\hline $445 \mathrm{~m}$ & 20100703 & $13: 08: 15$ & 180,90 & 14:08:55 & 225,315 & $\mathrm{~A}+, \mathrm{B}+$ \\
\hline $448 m$ & 20100705 & $21: 08: 15$ & 315 & 20:08:56 & 45 & $\mathrm{~A}+, \mathrm{B}-$ \\
\hline $449 m$ & 20100706 & 08:08:15 & 90,360 & 09:08:56 & 270 & $\mathrm{~A}--, \mathrm{B}+$ \\
\hline $464 m$ & 20100801 & 04:08:15 & 90 & 05:09:04 & 270 & $\mathrm{~A}-, \mathrm{B}--$ \\
\hline $465 \mathrm{a} \mathrm{m}$ & 20100801 & 09:08:15 & 90 & 10:09:04 & 315 & $\mathrm{~A}+, \mathrm{B}++$ \\
\hline $465 \mathrm{~b} \mathrm{~m}$ & 20100801 & 08:08:15 & 45 & 10:08:15 & 315 & $\mathrm{~A}-, \mathrm{B}-$ \\
\hline $470 \mathrm{~m}$ & 20100807 & $20: 08: 15$ & 90,225 & 21:09:05 & 270 & $\mathrm{~A}++, \mathrm{B}++, \mathrm{H}$ \\
\hline $471 \mathrm{~m}$ & 20100808 & 17:08:15 & 225 & 18:09:06 & 135 & $\mathrm{~A}+, \mathrm{B}+$ \\
\hline $474 m$ & 20100814 & $12: 08: 15$ & 135 & 13:09:07 & $225-270$ & $\mathrm{~A}+, \mathrm{B}+, \mathrm{H}$ \\
\hline $476 \mathrm{~m}$ & 20100815 & 14:08:15 & 45 & 16:09:07 & 315 & $\mathrm{~A}+, \mathrm{B}-$ \\
\hline $479 m$ & 20100818 & 06:08:15 & 45 & 07:09:08 & 315 & $\mathrm{~A}+, \mathrm{B}+$ \\
\hline $500 \mathrm{~m}$ & 20100911 & 06:08:15 & 45,225 & 07:09:12 & 315,135 & $\mathrm{~A}+, \mathrm{B}+$ \\
\hline $511 \mathrm{~m}$ & 20101001 & 05:08:15 & 315 & 06:09:14 & 45 & $\mathrm{~A}++, \mathrm{B}+$ \\
\hline $515 \mathrm{~m}$ & 20101006 & 10:08:15 & 90 & 14:09:14 & 270 & $\mathrm{~A}+, \mathrm{B}+$ \\
\hline $516 \mathrm{~m}$ & 20101007 & $15: 08: 15$ & 0 & 16:09:14 & 0 & $\mathrm{~A}+, \mathrm{B}+$ \\
\hline $519 m$ & 20101011 & 05:08:15 & 90 & 06:09:14 & 270 & $\mathrm{~A}++, \mathrm{B}++$ \\
\hline $525 \mathrm{~m}$ & 20101026 & $15: 08: 15$ & 135,315 & 16:09:14 & 225,0 & $\mathrm{~A}++, \mathrm{B}+$ \\
\hline $528 \mathrm{~m}$ & 20101028 & $16: 08: 15$ & 215 & 15:09:13 & 90 & $\mathrm{~A}++, \mathrm{B}++$ \\
\hline $546 \mathrm{~m}$ & 20101116 & 14:08:15 & 135 & 15:09:11 & 225 & $\mathrm{~A}+, \mathrm{B}-$ \\
\hline $551 \mathrm{~m}$ & 20101124 & 09:08:15 & 135 & 10:09:10 & 270 & $\mathrm{~A}-, \mathrm{B}+$ \\
\hline 552 & 20101126 & 00:08:15 & 225 & 01:09:10 & 135 & $\mathrm{~A}+, \mathrm{B}+$ \\
\hline 558 & 20101202 & 13:08:15 & 270 & 16:09:09 & 90 & $\mathrm{~A}-, \mathrm{B}-$ \\
\hline 561 & 20101206 & $00: 08: 15 n$ & 135 & 23:09:08 & 225 & $\mathrm{~A}++, \mathrm{B}+$ \\
\hline 566 & 20101212 & 08:08:15 & 45,135 & 10:09:06 & 225,315 & $\mathrm{~A}+, \mathrm{B}+, \mathrm{pH}$ \\
\hline 568 & 20101214 & 18:08:15 & 45 & 19:09:06 & 315 & $\mathrm{~A}+, \mathrm{B}+$ \\
\hline 574 & 20101223 & 12:08:15 & 90 & 13:09:04 & 270 & $\mathrm{~A}+, \mathrm{B}+$ \\
\hline 576 & 20101226 & 09:08:15 & 225 & 10:09:03 & 135 & $\mathrm{~A}++, \mathrm{B}++$ \\
\hline
\end{tabular}

One event was found in the synoptic movies subsequently, it was labeled with $465 \mathrm{~b}$ and the previously found event 465 with 465 a. 
Fit results of the modeled "Best-of" events:

Table 4 Fit results of the 51 modeled events which are listed in Table 3. No indicates the CME-Number in the COR2 list, the six following parameters are the same introduced in Section 2 and summarized in Table 1. The last column denotes the timestamp of the COR2 image used for fitting.

\begin{tabular}{|c|c|c|c|c|c|c|c|}
\hline No & $\begin{array}{l}\phi \\
{[\mathrm{deg}]}\end{array}$ & $\begin{array}{l}\theta \\
{[\operatorname{deg}]}\end{array}$ & $\begin{array}{l}\gamma \\
{[\mathrm{deg}]}\end{array}$ & $\begin{array}{l}h_{\text {front }} \\
r_{\text {sun }}\end{array}$ & $\kappa$ & $\begin{array}{l}\alpha \\
{[\operatorname{deg}]}\end{array}$ & $\begin{array}{l}\text { Time of modeling } \\
\text { yyyymmdd-hhmmss }\end{array}$ \\
\hline 325 & 39.132 & -18.446 & 15.091 & 16.786 & 0.277 & 23.198 & $20100201-210815$ \\
\hline 333 & 219.132 & -15.653 & -22.919 & 11.714 & 0.234 & 16.491 & $20100211-230815$ \\
\hline 337 & 197.888 & 11.180 & -27.392 & 12.214 & 0.330 & 17.888 & $20100214-050815$ \\
\hline 347 & 339.876 & -24.037 & -19.566 & 13.071 & 0.336 & 18.167 & $20100225-000815$ \\
\hline 350 & 144.223 & 36.335 & 1.118 & 11.714 & 0.517 & 28.229 & $20100228-180815$ \\
\hline 351 & 19.008 & -19.008 & 5.031 & 14.714 & 0.397 & 18.167 & $20100301-070815$ \\
\hline 359 & 48.074 & 12.857 & -13.415 & 13.143 & 0.333 & 18.726 & $20100309-110815$ \\
\hline 365 & 19.008 & -8.384 & 2.236 & 13.786 & 0.376 & 11.180 & $20100314-130815$ \\
\hline 369 & 106.211 & -11.740 & -6.709 & 13.714 & 0.385 & 18.726 & $20100319-200815$ \\
\hline 370 & 205.715 & 12.857 & -24.037 & 12.214 & 0.597 & 40.528 & $20100320-090815$ \\
\hline 373 & 21.244 & -1.118 & -39.690 & 15.000 & 0.244 & 9.783 & $20100326-170815$ \\
\hline 376 & 126.335 & 1.678 & 25.715 & 11.857 & 0.388 & 22.640 & $20100329-120815$ \\
\hline 377 & 253.789 & -28.510 & -16.771 & 15.000 & 0.410 & 14.533 & $20100330-140815$ \\
\hline 379 & 258.260 & -26.273 & -1.118 & 13.643 & 0.419 & 16.491 & $20100403-120815$ \\
\hline 383 & 195.653 & -1.678 & 8.383 & 14.928 & 0.367 & 16.211 & $20100408-080815$ \\
\hline 386 & 326.459 & -15.093 & 23.477 & 12.214 & 0.394 & 16.771 & $20100411-080815$ \\
\hline 387 & 266.087 & -25.155 & -29.630 & 13.429 & 0.244 & 9.783 & $20100412-110815$ \\
\hline 389 & 159.876 & 35.217 & -21.803 & 14.786 & 0.499 & 17.888 & $20100413-150815$ \\
\hline 391 & 91.678 & -21.242 & 12.857 & 13.357 & 0.514 & 15.932 & $20100419-040815$ \\
\hline 392 & 86.087 & 26.271 & -27.950 & 15.286 & 0.622 & 10.901 & $20100420-000815$ \\
\hline 404 & 251.554 & -24.597 & 90.000 & 15.500 & 0.665 & 11.460 & $20100506-020815$ \\
\hline 415 & 125.215 & -0.560 & -32.422 & 12.072 & 0.336 & 11.739 & $20100523-000815$ \\
\hline 416 & 320.868 & 7.826 & -9.506 & 12.857 & 0.480 & 13.975 & $20100523-220815$ \\
\hline 418 & 304.099 & -3.913 & -31.304 & 11.929 & 0.520 & 20.123 & $20100524-170815$ \\
\hline 426 & 319.752 & 2.236 & 28.510 & 14.429 & 0.333 & 26.553 & $20100604-160815$ \\
\hline 429 & 336.521 & 30.186 & -40.810 & 16.286 & 0.327 & 6.149 & $20100612-180815$ \\
\hline 432 & 228.074 & -30.186 & -7.267 & 14.571 & 0.351 & 24.317 & $20100615-050815$ \\
\hline 434 & 326.459 & 2.795 & -7.828 & 14.000 & 0.262 & 24.595 & $20100616-210815$ \\
\hline 435 & 98.381 & 20.124 & -23.477 & 13.214 & 0.311 & 18.726 & $20100619-040815$ \\
\hline 437 & 305.219 & 10.622 & 2.236 & 13.714 & 0.213 & 15.652 & $20100621-030815$ \\
\hline 444 & 346.583 & 10.062 & -22.919 & 14.928 & 0.428 & 14.255 & $20100703-080815$ \\
\hline 445 & 205.715 & -26.273 & 1.118 & 18.072 & 0.284 & 15.373 & $20100703-140815$ \\
\hline 448 & 320.868 & 40.806 & -40.250 & 12.643 & 0.434 & 13.975 & $20100705-210815$ \\
\hline 449 & 134.161 & -5.031 & -21.242 & 14.000 & 0.268 & 9.783 & $20100706-100815$ \\
\hline 464 & 102.856 & 7.267 & 36.335 & 12.000 & 0.127 & 11.739 & $20100801-060815$ \\
\hline $465 \mathrm{a}$ & 79.380 & 13.415 & -67.640 & 14.000 & 0.729 & 23.198 & $20100801-100815$ \\
\hline $465 b$ & 135.281 & 32.981 & -15.653 & 4.857 & 0.271 & 16.211 & $20100801-100815$ \\
\hline
\end{tabular}


Table 4 (Continued)

\begin{tabular}{llllllll}
\hline No & $\begin{array}{l}\phi \\
{[\mathrm{deg}]}\end{array}$ & $\begin{array}{l}\gamma \\
{[\mathrm{deg}]}\end{array}$ & $\begin{array}{l}\gamma \\
{[\mathrm{deg}]}\end{array}$ & $\begin{array}{l}h_{\text {front }} \\
r_{\text {sun }}\end{array}$ & $\kappa$ & $\begin{array}{l}\alpha \\
{[\mathrm{deg}]}\end{array}$ & $\begin{array}{l}\text { Time of modeling } \\
\text { yyyymmdd-hhmmss }\end{array}$ \\
\hline 470 & 324.223 & -1.118 & -32.422 & 21.857 & 0.539 & 8.663 & $20100807-210815$ \\
471 & 200.124 & -22.919 & 2.795 & 12.857 & 0.431 & 23.478 & $20100808-180815$ \\
474 & 353.293 & -5.031 & 3.353 & 14.357 & 0.816 & 31.025 & $20100814-120815$ \\
476 & 310.806 & 32.981 & -25.155 & 15.214 & 0.373 & 21.522 & $20100815-150815$ \\
479 & 348.818 & -6.709 & -43.043 & 16.071 & 0.717 & 12.019 & $20100818-070815$ \\
500 & 264.967 & 22.360 & 2.795 & 13.214 & 0.579 & 19.565 & $20100911-070815$ \\
511 & 176.645 & 42.484 & -1.118 & 14.714 & 0.299 & 22.919 & $20101001-050815$ \\
515 & 280.620 & 21.802 & -4.471 & 9.429 & 0.345 & 20.404 & $20101006-100815$ \\
516 & 8.942 & 45.279 & 23.477 & 13.214 & 0.357 & 37.174 & $20101007-150815$ \\
519 & 225.839 & 6.709 & -41.366 & 13.571 & 0.523 & 35.496 & $20101011-060815$ \\
525 & 69.318 & -25.715 & -40.810 & 13.357 & 0.545 & 11.739 & $20101026-140815$ \\
528 & 205.715 & -17.888 & 2.795 & 10.572 & 0.487 & 35.217 & $20101028-150815$ \\
546 & 86.087 & -25.715 & -2.795 & 12.857 & 0.382 & 19.286 & $20101116-150815$ \\
551 & 22.360 & -21.803 & 22.919 & 13.786 & 0.397 & 22.361 & $20101124-090815$ \\
\hline
\end{tabular}

\section{References}

Chen, J., Howard, R.A., Brueckner, G.E., Santoro, R., Krall, J., Paswaters, S.E., et al.: 1997, Evidence of an erupting magnetic flux rope: LASCO Coronal Mass Ejection of 1997 April 13. Astrophys. J. 490, $191-194$.

Cremades, H., Bothmer, V.: 2004, On the three-dimensional configuration of coronal mass ejections. Astron. Astrophys. 422, $307-322$.

Howard, R.A., Moses, J.D., Vourlidas, A., Newmark, J.S., Socker, D.G., Plunkett, S.P., et al.: 2008, Sun Earth Connection Coronal and Heliospheric Investigation (SECCHI). Space Sci. Rev. 136, 67-115.

Kaiser, M.L., Kucera, T.A., Davila, J.M., St. Cyr, O.C., Guhathakurta, M., Christian, E.: 2008, The STEREO mission: an introduction. Space Sci. Rev. 136, 5-16.

Robbrecht, E., Patsourakos, S., Vourlidas, A.: 2009, No Trace left behind: STEREO Observation of a Coronal Mass Ejection without low-coronal signatures. Astrophys. J. 701, 283-291.

St. Cyr, O.C., Howard, R.A., Sheeley, N.R. Jr., Plunkett, S.P., Michels, D.J., Paswaters, S.E., et al.: 2000, Properties of coronal mass ejections: SOHO LASCO observations from January 1996 to June 1998. J. Geophys. Res. 105, 18,169-18,185.

Thernisien, A.F.R., Howard, R.A., Vourlidas, A.: 2006, Modeling of flux rope coronal mass ejections. Astrophys. J. 652, $763-773$.

Thernisien, A.F.R., Vourlidas, A., Howard, R.A.: 2009, Forward modeling of coronal mass ejections using STEREO/SECCHI data. Solar Phys. 256, $111-130$.

Tripathi, D., Bothmer, V., Cremades, H.: 2004, The basic characteristics of EUV post-eruptive arcades and their role as tracers of coronal mass ejection source regions. Astron. Astrophys. 422, 337-349.

Vourlidas, A., Subramanian, P., Dere, K.P., Howard, R.A.: 2000, Large-angle spectrometric coronagraph measurements of the energetics of coronal mass ejections. Astrophys. J. 534, 456-467.

Yashiro, S., Gopalswamy, N., Michalek, G., St. Cyr, O.C., Plunkett, S.P., Rich, N.B., et al.: 2004, A catalog of white light coronal mass ejections observed by the SOHO spacecraft. J. Geophys. Res. 109(A07105), $1-11$. 